\title{
Classification of the glioma grading using radiomics analysis
}

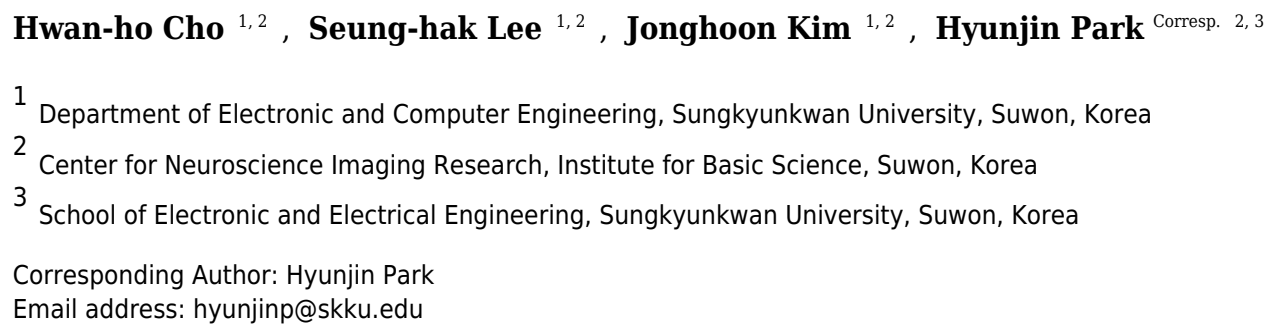

Background: Grading of gliomas is critical information related to prognosis and survival. We aimed to apply a radiomics approach using various machine learning classifiers to determine the glioma grading. Methods: We considered 285 (high grade $n=210$, low grade $n=75$ ) cases obtained from the Brain Tumor Segmentation 2017 Challenge. Manual annotations of enhancing tumors, non-enhancing tumors, necrosis, and edema were provided by the database. Each case was multi-modal with T1-weighted, T1-contrast enhanced, T2-weighted, and FLAIR images. A five-fold cross validation was adopted to separate the training and test data. A total of 468 radiomics features were calculated for three types of regions of interest. The minimum redundancy maximum relevance algorithm was used to select features useful for classifying glioma grades in the training cohort. The selected features were used to build three classifier models of logistics, support vector machines, and random forest classifiers. The classification performance of the models was measured in the training cohort using accuracy, sensitivity, specificity, and area under the curve (AUC) of the receiver operating characteristic curve. The trained classifier models were applied to the test cohort. Results: Five significant features were selected for the machine learning classifiers and the three classifiers showed an average AUC of 0.9400 for training cohorts and 0.9030 (logistic regression 0.9010 , support vector machine 0.8866 , and random forest 0.9213 ) for test cohorts. Discussion: Glioma grading could be accurately determined using machine learning and feature selection techniques in conjunction with a radiomics approach. The results of our study might contribute to high-throughput computer aided diagnosis system for gliomas. 


\section{Title: Classification of the glioma grading using radiomics analysis}

2

3 Authors: Hwan-ho Cho ${ }^{1,2}$, Seung-hak Lee ${ }^{1,2}$, Jonghoon Kim ${ }^{1,2}$, and Hyunjin Park ${ }^{2,3}$

$4{ }^{1}$ Department of Electronic and Computer Engineering, Sungkyunkwan University, Suwon,

5 Korea

$6{ }^{2}$ Center for Neuroscience Imaging Research, Institute for Basic Science, Suwon, Korea

$7 \quad{ }^{3}$ School of Electronic and Electrical Engineering, Sungkyunkwan University, Suwon, Korea

9 Address correspondence to:

10 Hyunjin Park, Ph.D.

11 Center for Neuroscience Imaging Research

12 School of Electronic and Electrical Engineering

13 Sungkyunkwan University, Suwon, 16419, Korea

14 Phone: +82-31-299-4956; Fax: +82-31-290-5819; Email: hyunjinp@skku.edu 


\section{Abstract}

16 Background: Grading of gliomas is critical information related to prognosis and survival. We

17 aimed to apply a radiomics approach using various machine learning classifiers to determine the 18 glioma grading.

19 Methods: We considered 285 (high grade $n=210$, low grade $n=75$ ) cases obtained from the Brain Tumor Segmentation 2017 Challenge. Manual annotations of enhancing tumors, non-enhancing tumors, necrosis, and edema were provided by the database. Each case was multi-modal with T1weighted, T1-contrast enhanced, T2-weighted, and FLAIR images. A five-fold cross validation was adopted to separate the training and test data. A total of 468 radiomics features were calculated for three types of regions of interest. The minimum redundancy maximum relevance algorithm was used to select features useful for classifying glioma grades in the training cohort. The selected features were used to build three classifier models of logistics, support vector machines, and random forest classifiers. The classification performance of the models was measured in the training cohort using accuracy, sensitivity, specificity, and area under the curve (AUC) of the receiver operating characteristic curve. The trained classifier models were applied to the test cohort.

31 Results: Five significant features were selected for the machine learning classifiers and the three 32 classifiers showed an average AUC of 0.9400 for training cohorts and 0.9030 (logistic regression 0.9010, support vector machine 0.8866 , and random forest 0.9213 ) for test cohorts.

Discussion: Glioma grading could be accurately determined using machine learning and feature selection techniques in conjunction with a radiomics approach. The results of our study might contribute to high-throughput computer aided diagnosis system for gliomas. 


\section{Introduction}

Gliomas are primary brain tumors arising from glial cells. The grades of gliomas have been determined based on histology according to the World Health Organization standard (Louis et al., 2007). Recently, revised criteria have been introduced that consider genetic factors such as isocitrate dehydrogenase mutation and 1p/19q codeletion (Louis et al., 2016). The grading of gliomas is critical information related to prognosis and survival (Wu et al., 2010; Louis et al., 2016).

A scheme that dichotomizes the graded gliomas into high-grade gliomas (HGG) and low-grade gliomas (LGG) has been widely adopted. It is important to differentiate HGG from LGG for assessing tumor progression and therapy planning (Louis et al., 2007). An experienced observer can differentiate between the two grades well based on tumor enhancement, but a computer algorithm might match the performance of the human expert with increased speed. More importantly, the computer algorithm might contribute to developing high-throughput computer aided diagnosis system.

An algorithm known as radiomics has recently emerged as a powerful methodology to quantify the characteristics of tumors in a non-invasive manner (Yip \& Aerts, 2016). Many studies have demonstrated that distinct tumor types in many organs can be quantified by radiomics analysis and the results of the radiomics can be used as imaging biomarkers for supporting clinical decision making (Zacharaki et al., 2009; Aerts et al., 2014; Kickingereder et al., 2016; Li et al., 2016; Bowen et al., 2017). Radiomics can also reveal novel characteristics of brain tumors, as demonstrated by a recent study (Zhou et al., 2017b). Many studies predicted the chemotherapeutic response and survival of patients with glioblastoma using a large number of imaging features based on MR imaging (Itakura et al., 2015; Cui et al., 2016; Kickingereder et al., 2016; Prasanna et al., 2016; Lao et al., 2017; Zhou et al., 2017a). Other studies have predicted prognosis using features obtained 
61 from functional imaging (Ryu et al., 2014; Lee et al., 2016). Recently, radiomics has been combined

62 with genomics to leverage two distinct types of information to better study various tumor types

63 (Gutman et al., 2015; Li et al., 2016; Beig et al., 2018; Zinn et al., 2018). The new approach is

64 referred to as radiogenomics and has to potential to reveal novel findings combining two distinct

65 high dimensional information of gene and imaging information. Many existing brain tumor studies

66 related to radiomics mainly focused on glioblastoma which is the most aggressive glioma and

67 considered a limited number of imaging modalities (Ryu et al., 2014; Itakura et al., 2015; Cui et al.,

68 2016; Lee et al., 2016). Multi-modal data are high-dimensional by nature and thus handling them

69 properly requires carefully chosen machine learning approaches. However, existing literature of

70 radiomics using multi-modal data and various machine learning approaches is relatively scarce.

71 In this paper, we applied a radiomics approach combined with various machine learning 72 approaches to multi-modal imaging of glioma patients to study whether the grade of glioma can be 73 determined noninvasively. The aim of this study was to quantify glioma with a radiomics approach 74 and to use the results to classify the gliomas as HGG or LGG. We used annotated multi-modal MRI 75 imaging data from a research database (Menze et al., 2015; Bakas et al., 2017a,b,c). A total of 468 76 quantitative radiomics were computed for four MRI modalities and three regions of interest (ROIs).

77 Significant features were selected using relevance and redundancy criteria. We aimed to 78 demonstrate the effectiveness of these features on the classification of each glioma's 79 histopathological grade using three machine learning classifiers. The overall workflow of this paper 80 is shown in Figure 1.

$81<$ Figure 1> 
84

85

86

87

88

89

90

91

\section{Patients and imaging}

The institutional review broad (IRB) of Sungkyunkwan University approved our study (IRB\# 2015-09-007). Consent was waived for this retrospective study. Our study was performed in full accordance with local IRB guidelines. We considered data from the MICCAI Brain Tumor Segmentation 2017 Challenge (BraTS 2017) (Menze et al., 2015; Bakas et al., 2017a,b,c). This dataset was derived from pre-operative baseline scans from two variants of the Cancer Imaging Archive (TCIA) (Clark et al., 2013), the TCIA-glioblastoma (GBM) and TCIA-LGG collections (Pedano et al., 2016; Scarpace et al., 2016). Each is a multi-institutional data mix from eight and five institutions, respectively. Detailed patient and scanner information can be found in the data citation (Bakas et al., 2017a, c,b). We considered 210 HGG and 75 LGG patients. HGG included glioblastoma multi-forme (GBM) and LGG included astrocytomas, oligodendroglioma, and oligoastrocytomas (Pedano et al., 2016; Scarpace et al., 2016; Bakas et al., 2017a).

Each patient had pre-operative images in four modalities (T1, T1-contrast enhanced, T2, FLAIR). All images were preprocessed using the FMRIB Software Library (FSL). Each image was registered onto the common space (Rohlfing et al., 2010) and interpolated to a 1x1x1mm isotropic voxel grid. In addition, manual segmentations of enhancing tumors, non-enhancing tumors, necrosis, and edema in each image were also provided by the challenge organizers (Bakas et al., 2017a, c,b). Manual segmentation was performed using a semi-automatic method with expert confirmation (Bakas et al., 2017a). These specific preprocessing choices were made by the BraTS organizational committee (Menze et al., 2015; Bakas et al., 2017a). Our study was a single source study (just from the BraTS database) and thus we adopted a five-fold cross validation to separate the training and test cohorts to reduce overfitting. Each fold had a similar ratio of HGG and LGG. 
106 The ratio of HGG and LGG was maintained between the training and test sets. Table 1 contains

107 institutional information for all patients.

$108<$ Table $1>$

109

110 Tumor regions of interest

111 We combined the three manual segmentation results provided by BraTS into ROIs to extract multi-

112 regional radiomics features. We intended to obtain information from multiple tissue types rather

113 than single tissue type (Zacharaki et al., 2009). The first region (ROI type I) was created by merging

114 the non-enhancing tumor and necrotic region and the second region (ROI type II) was created by

115 adding the tumor region with enhancement to the first region. The third region (ROI type III)

116 combined the second region with the area of edema. The first region is the smallest region and the

117 third region is the largest region inclusive of multiple compartments. Representative ROIs are

118 shown in Figure 2.

$119<$ Figure 2>

120

121 Radiomics features

122 To compute high-dimensional imaging information needed for the radiomics approach, imaging

123 features were calculated using all three ROIs in four modalities from 3D volume. The features were

124 computed using a combination of open source code (Van Griethuysen et al., 2017) and in-house

125 generated computer code implemented in MATLAB (Mathworks Inc. Natick, MA, USA). For most

126 features, we used the open source software PyRadiomics so that the results could be easily

127 reproduced. For the features not available in PyRadiomics, we used our in-house MATLAB code 
128 which is provided as a supplement material. We computed a total of 468 radiomics features per 129 patient. We computed 24 shape-based (eight for each ROI), 228 histogram-based, and 216 texture130 based (192 gray-level co-occurrence matrix [GLCM] based and 24 intensity size-zone [ISZ] matrix131 based) features quantifying different characteristics of the tumor (Haralick, Shanmugam \& 132 Dinstein, 1973; Tixier et al., 2011; Davnall et al., 2012; Grove et al., 2015). The histogram-based 133 features were computed from 128 bin histogram computed over the whole intensity range. For the 134 GLCM features, we binned intensities with 128 bins. A total of 26 matrices corresponding to 26 3D directions with offset one were computed and then averaged to yield one matrix. The averaged matrix was used to compute GLCM features. For the ISZM features, we constructed a 128x256 matrix where the first dimension is binned intensity and the second dimension was size. The size was not quantized and if a blob was larger than 256 voxels, it was considered as a blob with size 256. We considered six neighbors (four in-plane and two out-of-plane ones) for defining the size of the blob. More details can be found in the supplement.

\section{Feature selection}

Feature values of the training cohort were normalized to z-scores for each feature across subjects.

Different radiomics features have different units and range. Some features were designed to fall between 0 and 1, while others have a very large range. All the features were subjected to the feature selection procedure. Without feature normalization, some features might be assigned a larger weight, while others might be assigned a lower weight depending on the distribution of feature values during the feature selection. Thus, we applied z-score normalization to the feature values, making the range of each feature relatively uniform. A similar approach can be found in another work (Kickingereder et al., 2016). We selected image features which could distinguish between 
151 HGG and LGG using the minimum redundancy maximum relevance (mRMR) algorithm (Peng,

152 Long \& Ding, 2005). The mRMR is described with three equations. The first equation (1) searches

153 for a set of features that maximizes the relevance $(\boldsymbol{D})$, where $\boldsymbol{S}$ is the total feature set, $\boldsymbol{c}$ is the grade

154 of glioma, $x_{i}$ is the individual feature, and $\boldsymbol{I}$ is the information measure. The second equation (2)

155 searches for a set of features that minimizes redundancy $(\boldsymbol{R})$ among the selected features, where $x_{i}$,

$156 x_{j}$ are the individual features. Equation (2) suppresses overlapping information among the selected

157 features. The previous steps are combined into the third equation (3), where the mRMR algorithm

158 identifies the optimal set of features that encourage maximum relevance and minimum redundancy.

159 We performed mRMR with mutual information as the information measure and selected the top

160 five features. We chose to select five features because we sought a compact model to distinguish

161 between HGG and LGG images.

$$
\begin{gathered}
\max D(S, c), D=\frac{1}{|S|} \sum_{x_{i} \in S} I\left(x_{i} ; c\right) . \\
\min R(S), R=\frac{1}{|S|^{2}} \sum_{x_{i}, x_{j} \in S} I\left(x_{i}, x_{j}\right) . \\
\max \Phi(D, R), \Phi=D-R .
\end{gathered}
$$

\section{Training the classification model}

A five-fold cross-validation was adopted as mentioned before and the classifiers were trained using the training fold only. We adopted three classifiers to demonstrate the effectiveness of the chosen features. Logistic regression ( $\mathrm{Ng} \&$ Jordan, 2002), support vector machine (SVM) (Cortes \& 
168 between HGG and LGG images. The logistic classifier fits the distribution of data to the binomial 169 distribution and provides a category related output with values between 0 and 1 . The SVM is trained

170 to maximize the margins of the plane separating the two categories in the feature space and is the

171 most common classifier used for binary classification. RF is an ensemble classifier composed of a

172 number of decision trees and it could lessen overfitting by training each decision tree using only a

173 subset of the entire data. To train the logistic regression classifier, selected radiomics features were

174 linearly regressed to binarized grades of glioma and then a radiomics score was constructed using

175 a linear combination of regression coefficients and feature values. The score was fitted to the

176 logistic model and a fixed threshold ( = .5) was applied to distinguish between HGG and LGG.

177 The cost function of the SVM was Lagrangian of the sum of the distance from each feature point 178 to the marginal line. We chose a linear kernel for the SVM. The selected features from the feature 179 selection step were vectorized and referred to as the 5D feature space. As a result, training of the 180 SVM was performed using the 5D feature space. The RF model was also trained using the same 181 information as the SVM and 200 decision trees were used for consensus result in the training step.

Applying the model to the test cohort and statistics

184

185

We applied the trained models to the test cohort. The selected features and the associated coefficients from the training step were applied to the test cohort. The actual values of the features were computed from the test cohort. Performance of both cohorts was measured using the accuracy, sensitivity, specificity, and area under the curve (AUC) value of the receiver operating characteristic (ROC) curve. The positive case for the confusion matrix was set to HGG. The adjusted R-squared value and p-value were calculated to evaluate the degree of fit of the model. As we adopted a fivefold cross-validation, we repeated the procedures of feature selection, model training, and testing 
191 steps five times each time leaving a different test fold out. Each left out fold led to one set of

192 performance measures, and thus we reported the average value of five measures. All analyses and 193 evaluation procedures were performed using MATLAB (Mathworks Inc. Natick, MA, USA)

\section{Results}

196

197

198

199

200

201

202

203

204

205

206

207

208

209

210

211

\section{Selected features from training}

The top five features from the mRMR feature selection algorithm were chosen as significant radiomics features from each fold. Table 2 shows the most stable four radiomics features which were selected at least three times from the five-fold cross-validation. Regarding the category of features, the morphological property of tumor was most effective at discriminating HGG from LGG. Especially, spherical disproportion, which indicated how much the tumor shape was distorted from an ideal sphere, was found to be most valuable. The next most efficacious features were the GLCM features, which represent texture characteristic of the intra-tumoral area. Regarding the imaging modality, one was from T1 contrast enhanced image, and the other was from FLAIR. Regarding the type of ROIs, one was from ROI type I (non-enhancing tumor and necrotic region) and the other three were from ROI type II (enhancing, non-enhancing tumor and necrotic region).

$<$ Table 2> 
212 Training performance of the three classifiers is shown in Table 3. Each performance value was

213 calculated by averaging the five-fold cross validation results. The RF classifier showed the best

214 training performance and three classifiers had an AUC of 0.9400 on average, which showed that

215 the classifiers were very successful at modeling the training cohort. The accuracy, sensitivity, and

216 specificity were measured as $0.9292,0.9786$, and 0.7911 on average. Figure 3 shows ROCs for the

217 three classifiers for all five folds.

$218<$ Table $3>$

$219<$ Figure $3>$

220

221 Model performance in test step

222 Table 4 shows the results of applying the model constructed at the training stage to the test cohort.

223 These results were obtained by fixing the image features selected by mRMR and all the model

224 parameters. The actual feature values were computed from the test cohort. Same as the training

225 phase, the RF classifier had the best performance with AUC 0.9213, and the average AUC of the

226 three classifiers was 0.9030 . The other performance measures were obtained in the same manner as

227 the training phase. The average accuracy, sensitivity, and specificity were $0.8854,0.9508$ and

2280.7022 , respectively. Figure 4 shows ROCs for the three classifiers in the test cohort for all five

229 folds.

$230<$ Table $4>$

$231<$ Figure 4>

232 


\section{Discussion}

235

236

237

238

239

240

241

242

243

244

245

246

247

248

249

250

251

252

253

254

255

A radiomics approach can compute high-dimensional features from in vivo imaging modalities, which in turn were used to differentiate between HGG and LGG in this study. Our radiomics approach was tested with three classifiers and we obtained a high average AUC value of 0.9030 in the test cohort. Particularly, the RF classifier showed the best performance with AUC 0.9213 for the test cohort. Our main contribution was to fully leverage available multi-modal imaging with various machine learning approaches to distinguish between LGG and HGG.

Others also attempted to differentiate HGG from LGG. Law et al. used relative cerebral blood volume measurements and metabolite ratios from proton MR spectroscopy, which resulted in a sensitivity of 0.950 and a specificity of 0.575 in discrimination between HGG and LGG (Law et al., 2003). A recent study applied conventional image processing approaches to a small scale data of 42 patients' processed perfusion MRI and achieved a sensitivity of 0.966 , specificity of 0.812 and AUC of 0.95 (Togao et al., 2016). Zacharaki et al. adopted texture features similar as our approach to differentiate between LGG and HGG (Zacharaki et al., 2009). They achieved an accuracy of 0.878 and AUC of 0.896 using SVM based recursive feature elimination (SVM-RFE) with a leave-one-out cross validation. The leave-one-out cross validation portion of that study might lead to overfitting, while our study could reduce the overfitting issue with a five-fold cross validation. In addition, SVM-RFE approach is tailored for the SVM classifier and thus applying the SVM-RFE in conjunction with other classifiers could lead to performance degradation.

We found four significant features that were stable through the five-fold cross validation. They were spherical disproportion of ROI type I (non-enhancing tumor and necrotic region), contrast of GLCM of ROI type II (enhancing, non-enhancing tumor, and necrotic region) from T1-contrast 
256 enhanced images, compactness of ROI type II, and autocorrelation of GLCM of ROI type II from

257 FLAIR images (Table 2). The spherical disproportion and compactness measure how much an

258

259

260

261

262

263

264

265

266

267

268

269

270

271

272

273

274

275

276

277

278

ROI shape differs from a sphere. The former is a measure based on volume measurements, while the latter is based on surface measurements. For spherical disproportion, an ideal sphere has value one and the value increases as the shape differs from the sphere. For compactness, an ideal sphere has value 0.0531 (i.e., $1 / 6 \pi$ ) and the value decreases as the shape differs from the sphere. Glioma shape is a well-known factor associated with malignancy, as irregular tumor shape is often associated with higher malignancy and poor prognosis (Claes, Idema \& Wesseling, 2007). We found that the shape of both non-enhancing and enhancing portion of the tumor were important in determining the glioma grades. Another important predictor of tumor prognosis is intratumoral heterogeneity (McGranahan \& Swanton, 2015). We found two significant texture features: the contrast and autocorrelation of GLCM. The texture features quantify textural information within the ROI and can reveal patterns of intensity heterogeneity. The contrast of GLCM measures the local intensity variation of GLCM and autocorrelation of GLCM measures the magnitude of the fineness and coarseness of textural patterns. These texture features have often been identified as significant in other radiomics studies (Tixier et al., 2011; Davnall et al., 2012; Ganeshan et al., 2013; Grove et al., 2015; Bowen et al., 2017). One feature was from ROI type I (non-enhancing tumor and necrotic region) and the other three were from ROI type II, which included ROI type I plus the enhancing compartment. This confirmed that we need to consider both tumor core and the enhanced portion to evaluate the tumor grading.

There are related studies of gliomas using machine learning approaches. Kickingereder et al. estimated the progression-free and overall survival of GBM patients using T1, T1-contrast enhanced and FLAIR images (Kickingereder et al., 2016). They used principal component analysis 
279 (PCA) to develop radiomics signatures from high-dimensional features. PCA is effective at 280 reducing dimensionality, but its results are difficult to interpret. Itakura et al. computed 281 quantitative features from T1 images of GBM patients and found phenotypic clusters associated 282 with molecular pathway activity through consensus clustering (Itakura et al., 2015). The adopted 283 clustering was effective at demonstrating the association between imaging features and degree of 284 malignancy, but the results were still difficult to interpret. One study developed machine learning285 based prognostic imaging biomarkers of GBM images using multi-modal imaging, similar to our 286 study (Cui et al., 2016). They adopted the L1-norm regularization method to select significant 287 imaging features and predict overall survival (Tibshirani, 1996). In summary, our study was 288 designed to produce stable and interpretable results of radiomics analysis compared to existing ones.

289

290

291

292

Recently, a machine learning algorithm known as deep learning (DL) has become the go-to methodology to drastically enhance the performance of existing machine learning techniques (Lecun, Bengio \& Hinton, 2015). DL approaches have shown promise in tumor grading, diagnosis and prognosis prediction (Ertosun \& Rubin, 2015; Litjens et al., 2016; Lao et al., 2017; Li et al., 2017). DL approach does not require the researcher to specify a set of features a priori, but can implicitly learn the features relevant to the problem, and thus can be effective for radiomics research. DL requires many more training samples compared to conventional machine learning approaches and additional issues arise when fine-tuning many hyper-parameters. These issues are challenging and we plan to pursue DL approaches in the future.

Our study has several limitations. We used open source data originally designated for a segmentation challenge, so we could not control for all factors between LGG and HGG groups. This might have included bias in patient selection. Independent validation using data from another clinical site is missing. This might hinder the applicability of our approach to new data. There was 
302 a class imbalance between two classes. We thought that each class had enough samples for 303 statistical modeling. Still, the class imbalance issue might be alleviated by minority class 304 oversampling techniques. The ROIs were provided by the database and the reproducibility of the 305 ROI was not verified. World Health Organization recently announced a new tumor classification 306 system of the central nervous system (Louis et al., 2016). It breaks up the glioma into five grades 307 considering not only histological information but also isocitrate dehydrogenase mutation and $3081 \mathrm{p} / 19 \mathrm{q}$ codeletion. Updated grading of gliomas was unavailable to us and thus we used the 309 information of the traditional grading system. Future studies should consider grading information 310 on the new grading system.

\section{Conclusions}

313 In conclusion, we showed that glioma grades could be accurately determined by a combination of 314 high dimensional imaging features, an advanced feature selection method and machine learning 315 classifiers. We believe the algorithm presented in our study might contribute to high-throughput 316 computer aided diagnosis system for gliomas. 


\section{Acknowledgments}

318 We would like to thank the organizers of the MICCAI Brain Tumor Segmentation 2017 Challenge. 


\section{References}

Aerts HJWL., Velazquez ER., Leijenaar RTH., Parmar C., Grossmann P., Cavalho S., Bussink J., Monshouwer R., Haibe-Kains B., Rietveld D., Hoebers F., Rietbergen MM., Leemans CR., Dekker A., Quackenbush J., Gillies RJ., Lambin P. 2014. Decoding tumour phenotype by noninvasive imaging using a quantitative radiomics approach. Nature Communications 5. DOI: $10.1038 /$ ncomms5006.

Bakas S., Akbari H., Sotiras A., Bilello M., Rozycki M., Kirby JS., Freymann JB., Farahani K., Davatzikos C. 2017a. Advancing The Cancer Genome Atlas glioma MRI collections with expert segmentation labels and radiomic features. Scientific Data 4:1-13. DOI: 10.1038/sdata.2017.117.

Bakas S., Akbari H., Sotiras A., Bilello M., Rozycki M., Kirby J., Freymann J., Farahani K., Davatzikos C. 2017b. Segmentation Labels and Radiomic Features for the Pre-operative Scans of the TCGA-LGG collection. The Cancer Imaging Archive. DOI: https://doi.org/10.7937/K9/TCIA.2017.GJQ7R0EF.

Bakas S., Akbari H., Sotiras A., Bilello M., Rozycki M., Kirby J., Freymann J., Farahani K., Davatzikos C. 2017c. Segmentation Labels and Radiomic Features for the Pre-operative Scans of the TCGA-GBM collection. The Cancer Imaging Archive. DOI: https://doi.org/10.7937/K9/TCIA.2017.KLXWJJ1Q.

Beig N., Patel J., Prasanna P., Hill V., Gupta A., Correa R., Bera K., Singh S., Partovi S., Varadan V., Ahluwalia M., Madabhushi A., Tiwari P. 2018. Radiogenomic analysis of hypoxia pathway is predictive of overall survival in Glioblastoma. Scientific Reports 8:111. DOI: $10.1038 / \mathrm{s} 41598-017-18310-0$.

Bowen SR., Yuh WTC., Hippe DS., Wu W., Partridge SC., Elias S., Jia G., Huang Z., Sandison GA., Nelson D., Knopp M V., Lo SS., Kinahan PE., Mayr NA. 2017. Tumor radiomic heterogeneity: Multiparametric functional imaging to characterize variability and predict response following cervical cancer radiation therapy. Journal of Magnetic Resonance Imaging:1-9. DOI: 10.1002/jmri.25874.

Breiman L. 2001. Random Forests. Machine Learning. DOI: 10.1023/A:1010933404324.

Claes A., Idema AJ., Wesseling P. 2007. Diffuse glioma growth: A guerilla war. Acta Neuropathologica 114:443-458. DOI: 10.1007/s00401-007-0293-7.

Clark K., Vendt B., Smith K., Freymann J., Kirby J., Koppel P., Moore S., Phillips S., Maffitt D., Pringle M., Tarbox L., Prior F. 2013. The cancer imaging archive (TCIA): Maintaining and operating a public information repository. Journal of Digital Imaging 26:1045-1057. DOI: 10.1007/s10278-013-9622-7.

Cortes C., Vapnik V. 1995. Support-Vector Networks. Machine Learning. DOI: 10.1023/A:1022627411411.

Cui Y., Tha KK., Terasaka S., Yamaguchi S., Wang J., Kudo K., Xing L., Shirato H., Li R. 2016. Prognostic Imaging Biomarkers in Glioblastoma: Development and Independent Validation on the Basis of Multiregion and Quantitative Analysis of MR Images. Radiology 278:546553. DOI: 10.1148/radiol.2015150358. 
360

361

362

363

364

365

366

367

368

369

370

371

372

373

374

375

376

377

378

379

380

381

382

383

384

385

386

387

388

389

390

391

392

393

394

395

396

397

398

399

400

Davnall F., Yip CSP., Ljungqvist G., Selmi M., Ng F., Sanghera B., Ganeshan B., Miles KA., Cook GJ., Goh V. 2012. Assessment of tumor heterogeneity: An emerging imaging tool for clinical practice? Insights into Imaging 3:573-589. DOI: 10.1007/s13244-012-0196-6.

Ertosun MG., Rubin DL. 2015. Automated Grading of Gliomas using Deep Learning in Digital Pathology Images: A modular approach with ensemble of convolutional neural networks. AMIA ... Annual Symposium proceedings / AMIA Symposium. AMIA Symposium 2015:1899-908. DOI: PMC4765616.

Ganeshan B., Goh V., Mandeville HC., Ng QS., Hoskin PJ., Miles KA. 2013. Non-small cell lung cancer: histopathologic correlates for texture parameters at CT. Radiology 266:326-36. DOI: $10.1148 /$ radiol.12112428.

Van Griethuysen JJM., Fedorov A., Parmar C., Hosny A., Aucoin N., Narayan V., Beets-Tan RGH., Fillion-Robin JC., Pieper S., Aerts HJWL. 2017. Computational radiomics system to decode the radiographic phenotype. Cancer Research 77:e104-e107. DOI: 10.1158/00085472.CAN-17-0339.

Grove O., Berglund AE., Schabath MB., Aerts HJWL., Dekker A., Wang H., Rios Velazquez E., Lambin P., Gu Y., Balagurunathan Y., Eikman E., Gatenby RA., Eschrich S., Gillies RJ. 2015. Quantitative computed tomographic descriptors associate tumor shape complexity and intratumor heterogeneity with prognosis in lung adenocarcinoma. PLOS ONE 10:1-14. DOI: $10.1371 /$ journal.pone.0118261.

Gutman DA., Dunn WD., Grossmann P., Cooper LAD., Holder CA., Ligon KL., Alexander BM., Aerts HJWL. 2015. Somatic mutations associated with MRI-derived volumetric features in glioblastoma. Neuroradiology. DOI: 10.1007/s00234-015-1576-7.

Haralick RM., Shanmugam K., Dinstein I. 1973. Textural Features for Image Classification. IEEE Transactions on Systems, Man, and Cybernetics SMC-3:610-621. DOI: 10.1109/TSMC.1973.4309314.

Itakura H., Achrol AS., Mitchell LA., Loya JJ., Liu T., Westbroek EM., Feroze AH., Rodriguez S., Echegaray S., Azad TD., Yeom KW., Napel S., Rubin DL., Chang SD., Harsh GR., Gevaert O. 2015. Magnetic resonance image features identify glioblastoma phenotypic subtypes with distinct molecular pathway activities. Science Translational Medicine 7:1-11. DOI: $10.1126 /$ scitranslmed.aaa7582.

Kickingereder P., Götz M., Muschelli J., Wick A., Neuberger U., Shinohara RT., Sill M., Nowosielski M., Schlemmer HP., Radbruch A., Wick W., Bendszus M., Maier-Hein KH., Bonekamp D. 2016. Large-scale radiomic profiling of recurrent glioblastoma identifies an imaging predictor for stratifying anti-angiogenic treatment response. Clinical Cancer Research 22:5765-5771. DOI: 10.1158/1078-0432.CCR-16-0702.

Lao J., Chen Y., Li Z-C., Li Q., Zhang J., Liu J., Zhai G. 2017. A Deep Learning-Based Radiomics Model for Prediction of Survival in Glioblastoma Multiforme. Scientific Reports. DOI: 10.1038/s41598-017-10649-8.

Law M., Yang S., Wang H., Babb JS., Johnson G., Cha S., Knopp EA., Zagzag D. 2003. Glioma Grading: Sensitivity, Specificity, and Predictive Values of Perfusion MR Imaging and Proton MR Spectroscopic Imaging Compared with Conventional MR Imaging. American 
401

402

403

404

405

406

407

408

409

410

411

412

413

414

415

416

417

418

419

420

421

422

423

424

425

426

427

428

429

430

431

432

433

434

435

436

437

438

439

440

441

Journal of Neuroradiology 24:1989-1998.

Lecun Y., Bengio Y., Hinton G. 2015. Deep learning. Nature 521:436-444. DOI: 10.1038 /nature 14539 .

Lee J., Jain R., Khalil K., Griffith B., Bosca R., Rao G., Rao A. 2016. Texture feature ratios from relative CBV maps of perfusion MRI are associated with patient survival in glioblastoma. American Journal of Neuroradiology 37:37-43. DOI: 10.3174/ajnr.A4534.

Li Z., Wang Y., Yu J., Guo Y., Cao W. 2017. Deep Learning based Radiomics (DLR) and its usage in noninvasive IDH1 prediction for low grade glioma. Scientific Reports 7:1-11. DOI: $10.1038 / \mathrm{s} 41598-017-05848-2$.

Li H., Zhu Y., Burnside ES., Drukker K., Hoadley KA., Fan C., Conzen SD., Whitman GJ., Sutton EJ., Net JM., Ganott M., Huang E., Morris EA., Perou CM., Ji Y., Giger ML. 2016. MR Imaging Radiomics Signatures for Predicting the Risk of Breast Cancer Recurrence as Given by Research Versions of MammaPrint, Oncotype DX, and PAM50 Gene Assays. Radiology 281:382-391. DOI: 10.1148/radiol.2016152110.

Litjens G., Sánchez CI., Timofeeva N., Hermsen M., Nagtegaal I., Kovacs I., Hulsbergen-Van De Kaa C., Bult P., Van Ginneken B., Van Der Laak J. 2016. Deep learning as a tool for increased accuracy and efficiency of histopathological diagnosis. Scientific Reports 6. DOI: $10.1038 /$ srep26286.

Louis DN., Ohgaki H., Wiestler OD., Cavenee WK., Burger PC., Jouvet A., Scheithauer BW., Kleihues P. 2007. The 2007 WHO classification of tumours of the central nervous system. Acta Neuropathologica 114:97-109. DOI: 10.1007/s00401-007-0243-4.

Louis DN., Perry A., Reifenberger G., Deimling A Von., Figarella D., Webster B., Hiroko KC., Wiestler OD., Kleihues P., Ellison DW. 2016. The 2016 World Health Organization Classification of Tumors of the Central Nervous System : a summary. Acta Neuropathologica 131:803-820. DOI: 10.1007/s00401-016-1545-1.

McGranahan N., Swanton C. 2015. Biological and therapeutic impact of intratumor heterogeneity in cancer evolution. Cancer Cell 27:15-26. DOI: 10.1016/j.ccell.2014.12.001.

Menze BH., Jakab A., Bauer S., Kalpathy-Cramer J., Farahani K., Kirby J., Burren Y., Porz N., Slotboom J., Wiest R., Lanczi L., Gerstner E., Weber MA., Arbel T., Avants BB., Ayache N., Buendia P., Collins DL., Cordier N., Corso JJ., Criminisi A., Das T., Delingette H., Demiralp Ç., Durst CR., Dojat M., Doyle S., Festa J., Forbes F., Geremia E., Glocker B., Golland P., Guo X., Hamamci A., Iftekharuddin KM., Jena R., John NM., Konukoglu E., Lashkari D., Mariz JA., Meier R., Pereira S., Precup D., Price SJ., Raviv TR., Reza SMS., Ryan M., Sarikaya D., Schwartz L., Shin HC., Shotton J., Silva CA., Sousa N., Subbanna NK., Szekely G., Taylor TJ., Thomas OM., Tustison NJ., Unal G., Vasseur F., Wintermark M., Ye DH., Zhao L., Zhao B., Zikic D., Prastawa M., Reyes M., Van Leemput K. 2015. The Multimodal Brain Tumor Image Segmentation Benchmark (BRATS). IEEE Transactions on Medical Imaging 34:1993-2024. DOI: 10.1109/TMI.2014.2377694.

Ng A., Jordan MI. 2002. On generative vs. discriminative classifiers: A comparison of logistic regression and naive bayes. Proceedings of Advances in Neural Information Processing. DOI: $10.1007 / \mathrm{s} 11063-008-9088-7$. 
442

443

444

445

446

447

448

449

450

451

452

453

454

455

456

457

458

459

460

461

462

463

464

465

466

467

468

469

470

471

472

473

474

475

476

477

478

479

480

481

Pedano N., Flanders AE., Scarpace L., Mikkelsen T., Eschbacher JM., Hermes B., Ostrom Q. 2016. Radiology Data from The Cancer Genome Atlas Low Grade Glioma [TCGA-LGG] collection. The Cancer Imaging Archive. DOI: ttp://doi.org/10.7937/K9/TCIA.2016.L4LTD3TK.

Peng H., Long F., Ding C. 2005. Feature selection based on mutual information: Criteria of MaxDependency, Max-Relevance, and Min-Redundancy. IEEE Trans. on Pattern Analysis and Machine Intelligence 27:1226-1238. DOI: 10.1109/TPAMI.2005.159.

Prasanna P., Patel J., Partovi S., Madabhushi A., Tiwari P. 2016. Radiomic features from the peritumoral brain parenchyma on treatment-naive multi-parametric MR imaging predict long versus short-term survival in glioblastoma multiforme: Preliminary findings. Eur Radiol. DOI: 10.1007/s00330-016-4637-3.

Rohlfing T., Zahr NM., Sullivan E V., Pfefferbaum A. 2010. The SRI24 multichannel atlas of normal adult human brain structure. Human Brain Mapping 31:798-819. DOI: 10.1002/hbm.20906.

Ryu YJ., Choi SH., Park SJ., Yun TJ., Kim JH., Sohn CH. 2014. Glioma: Application of wholetumor texture analysis of diffusion-weighted imaging for the evaluation of tumor heterogeneity. PLOS ONE 9. DOI: 10.1371/journal.pone.0108335.

Scarpace L., Mikkelsen T., Cha S., Rao S., Tekchandani S., Gutman D. 2016. Radiology Data from The Cancer Genome Atlas Glioblastoma Multiforme [TCGA-GBM] collection. The Cancer Imaging Archive. DOI: http://doi.org/10.7937/K9/TCIA.2016.RNYFUYE9.

Tibshirani R. 1996. Regression Selection and Shrinkage via the Lasso. Journal of the Royal Statistical Society B 58:267-288. DOI: 10.2307/2346178.

Tixier F., Le Rest CC., Hatt M., Albarghach N., Pradier O., Metges J-P., Corcos L., Visvikis D. 2011. Intratumor Heterogeneity Characterized by Textural Features on Baseline 18F-FDG PET Images Predicts Response to Concomitant Radiochemotherapy in Esophageal Cancer. Journal of Nuclear Medicine 52:369-378. DOI: 10.2967/jnumed.110.082404.

Togao O., Hiwatashi A., Yamashita K., Kikuchi K., Mizoguchi M., Yoshimoto K., Suzuki SO., Iwaki T., Obara M., Van Cauteren M., Honda H. 2016. Differentiation of high-grade and low-grade diffuse gliomas by intravoxel incoherent motion MR imaging. Neuro-Oncology 18:132-141. DOI: 10.1093/neuonc/nov147.

Wu W., Lamborn KR., Buckner JC., Novotny PJ., Chang SM., O'Fallon JR., Jaeckle KA., Prados MD. 2010. Joint NCCTG and NABTC prognostic factors analysis for high-grade recurrent glioma. Neuro-Oncology 12:164-172. DOI: 10.1093/neuonc/nop019.

Yip SSF., Aerts HJWL. 2016. Applications and limitations of radiomics. Physics in Medicine and Biology 61:R150-R166. DOI: 10.1088/0031-9155/61/13/R150.

Zacharaki EI., Wang S., Chawla S., Soo D. 2009. Classification of brain tumor type and grade using MRI texture and shape in a machine learning scheme. Magnetic Resonance in Medicine: An Official Journal of the International Society for Magnetic Resonance in Medicine 62:1609-1618. DOI: 10.1002/mrm.22147.Classification.

Zhou M., Chaudhury B., Hall LO., Goldgof DB., Gillies RJ., Gatenby RA. 2017a. Identifying 
482 spatial imaging biomarkers of glioblastoma multiforme for survival group prediction. Journal of Magnetic Resonance Imaging. DOI: 10.1002/jmri.25497. Cramer J., Napel S., Gillies R., Gevaert O., Gatenby R. 2017b. Radiomics in Brain Tumor: Image Assessment, Quantitative Feature Descriptors, and Machine-Learning Approaches. American Journal of Neuroradiology. DOI: 10.3174/ajnr.A5391.

Zinn PO., Singh SK., Kotrotsou A., Hassan I., Thomas G., Luedi MM., Elakkad A., Elshafeey N., Idris T., Mosley J., Gumin J., Fuller GN., DeGroot JF., Baladandayuthapani V., Sulman EP., Kumar AJ., Sawaya R., Lang FF., Piwnica-Worms D., Colen RR. 2018. A co-clinical radiogenomic validation study-Conserved magnetic resonance radiomic appearance of Periostin expressing Glioblastoma in patients and xenograft models. Clinical Cancer 
495

496

497

498

499

500

501

502

503

504

505

506

507

508

509

510

511

512

513

514

\section{Figure Caption}

Figure 1. Overall workflow of the study.

Figure 2. Examples of three types of ROIs used in our study. (a) T1 data. (b) ROI associated with T1. (c) T1C data. (d) ROI associated with T1C. (e) T2 data. (f) ROI associated with T2. (g) FLAIR data. (h) ROI associated with FLAIR. The left column (a) (c) (e) (g) shows different imaging modalities. The right column (b) (d) (f) (h) shows associated ROIs. The ROIs were specified in $3 \mathrm{D}$ but $2 \mathrm{D}$ representative examples are given. ROIs are visualized in the right column. Red indicates non-enhancing tumor and necrosis (ROI type I), yellow indicates enhancing tumor (ROI type II) and blue indicates edema (ROI type III) in the right column. T1; T1-weighted image, T2; T1C; T1-contrast enhanced; T2-weighted image, FLAIR; Fluid-Attenuated Inversion Recovery.

Figure 3. Performance curves of the five-fold cross validation in the training phase. (a) shows the ROC for the logistic regression classifier. (b) shows the ROC for the SVM classifier. (c) shows the ROC for the RF classifier.

Figure 4. Performance curves of the five-fold cross validation in the test phase. (a) shows the ROC for the logistic regression classifier. (b) shows the ROC for the SVM classifier. (c) shows the ROC for the RF classifier. 
Table $\mathbf{1}$ (on next page)

Institutional information of patients (Bakas et al., 2017a)

TCGA; The tumor genome atlas. 


\begin{tabular}{|c|c|c|}
\hline Collection & Institutions & TCGA ID \\
\hline \multirow{8}{*}{ TCGA-GBM } & Henry Ford Hospital, Detroit, MI & TCGA-06 \\
\hline & CWRU School of Medicine, Cleveland, $\mathrm{OH}$ & TCGA-19 \\
\hline & University of California, San Francisco, CA & TCGA-08 \\
\hline & Emory University, Atlanta, GA & TCGA-14 \\
\hline & MD Anderson Cancer Center, Houston, TX & TCGA-02 \\
\hline & Duke University School of Medicine, Durham, NC & TCGA-12 \\
\hline & Thomas Jefferson University, Philadelphia, PA & TCGA-76 \\
\hline & $\begin{array}{l}\text { Fondazione IRCCS } \\
\text { Instituto Neuroligico C. Besta, Milan, Italy }\end{array}$ & TCGA-27 \\
\hline \multirow{5}{*}{ TCGA-LGG } & St Joseph Hospital/Medical Center, Phoenix, AZ & TCGA-HT \\
\hline & Henry Ford Hospital, Detroit, MI & TCGA-DU \\
\hline & Case Western Reserve University, Cleveland, $\mathrm{OH}$ & TCGA-FG \\
\hline & Thomas Jefferson University, Philadelphia, PA & TCGA-CS \\
\hline & University of North Carolina, Chapel Hill, NC & TCGA-EZ \\
\hline
\end{tabular}

1 TCGA; The tumor genome atlas. 
Table 2 (on next page)

Selected features via mRMR based on stability over five folds. 
1

\begin{tabular}{|c|c|c|c|c|}
\hline & Feature name & Modality & Category & ROI type \\
\hline 1 & Spherical Disproportion & Shape & Shape & 1 \\
\hline 2 & Contrast & T1c & GLCM & 2 \\
\hline 3 & Compactness & Shape & Shape & 2 \\
\hline 4 & Autocorrelation & FLAIR & GLCM & 2 \\
\hline
\end{tabular}

2 


\section{Table 3(on next page)}

Training performance measures using various classifiers

Note: Each performance value was calculated by averaging the results of the five-fold cross validation. SVM; support vector machine, RF; random forest, AUC; area under the curve 
1

\begin{tabular}{|c|c|c|c|c|c|c|}
\hline Classifier & Accuracy & Sensitivity & Specificity & AUC & $\begin{array}{c}\text { Adjusted } \\
\text { R-squared }\end{array}$ & p-value \\
\hline Logistic & 0.8895 & 0.9643 & 0.6800 & 0.9066 & 0.4877 & $8.0686 \mathrm{e}-23$ \\
\hline SVM & 0.8983 & 0.9714 & 0.6933 & 0.9135 & 0.4461 & $6.5597 \mathrm{e}-13$ \\
\hline RF & 1 & 1 & 1 & 1 & 0.9537 & $7.4280 \mathrm{e}-148$ \\
\hline Average & 0.9292 & 0.9786 & 0.7911 & 0.9400 & & \\
\hline
\end{tabular}

2 Note: Each performance value was calculated by averaging the results of the five-fold cross

3 validation. SVM; support vector machine, RF; random forest, AUC; area under the curve 


\section{Table 4 (on next page)}

Test performance measures using various classifiers.

Note: Each performance value was calculated by averaging the results of the five-fold cross validation. SVM; support vector machine, RF; random forest, AUC; area under the curve 
1

\begin{tabular}{|c|c|c|c|c|c|c|}
\hline Classifier & Accuracy & Sensitivity & Specificity & AUC & $\begin{array}{c}\text { Adjusted } \\
\text { R-squared }\end{array}$ & p-value \\
\hline Logistic & 0.8877 & 0.9619 & 0.6800 & 0.9010 & 0.4882 & $5.6693 \mathrm{e}-23$ \\
\hline SVM & 0.8807 & 0.9476 & 0.6933 & 0.8866 & 0.3989 & $4.2893 \mathrm{e}-05$ \\
\hline RF & 0.8877 & 0.9429 & 0.7333 & 0.9213 & 0.5725 & $2.4653 \mathrm{e}-10$ \\
\hline Average & 0.8854 & 0.9508 & 0.7022 & 0.9030 & & \\
\hline
\end{tabular}

2 Note: Each performance value was calculated by averaging the results of the five-fold cross

3 validation. SVM; support vector machine, RF; random forest, AUC; area under the curve 


\section{Figure 1}

Overall workflow of the study.

Image Acquisition

\& ROI specification

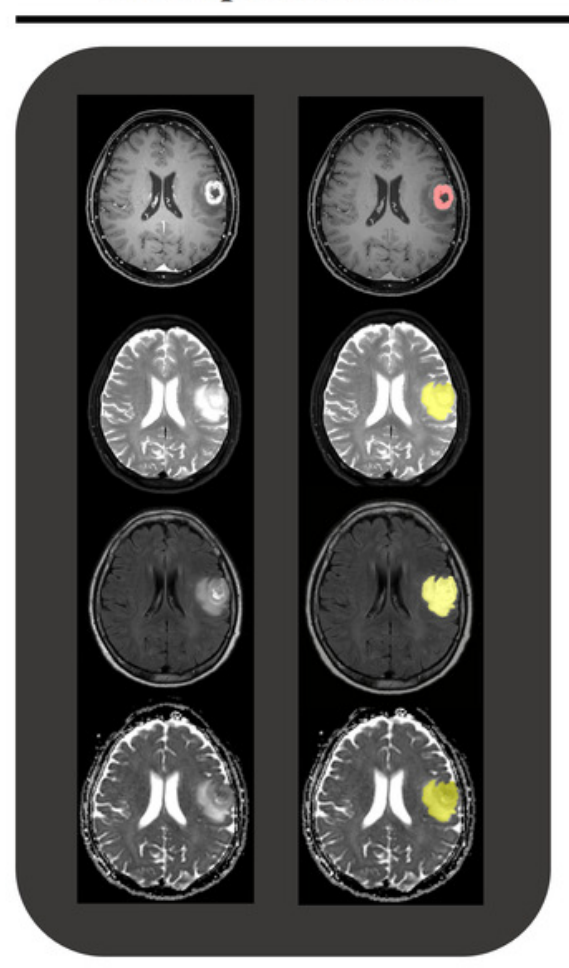

Radiomics Feature Extraction
Model Training
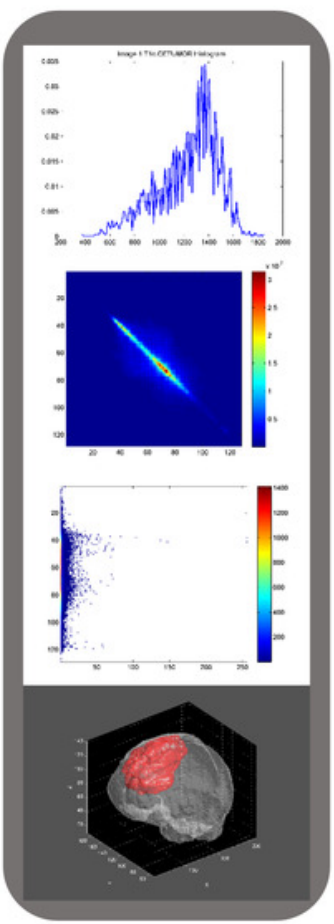

Logistic Classifier Support Vector Machine Random Forest
Performance Testing
- Accuracy

- Sensitivity

- Specificity

- ROC curve (AUC)

- Adjusted Rsquared

- p-value 


\section{Figure 2}

Examples of three types of ROls used in our study.

(a) T1 data. (b) ROI associated with T1. (c) T1C data. (d) ROI associated with T1C. (e) T2 data.

(f) ROI associated with T2. (g) FLAIR data. (h) ROI associated with FLAIR. The left column (a)

(c) (e) (g) shows different imaging modalities. The right column (b) (d) (f) (h) shows

associated ROls. The ROls were specified in 3D but 2D representative examples are given.

ROIs are visualized in the right column. Red indicates non-enhancing tumor and necrosis (ROI type I), yellow indicates enhancing tumor (ROI type II) and blue indicates edema (ROI type III) in the right column. T1; T1-weighted image, T2; T1C; T1-contrast enhanced; T2-weighted image, FLAIR; Fluid-Attenuated Inversion Recovery. 
T1

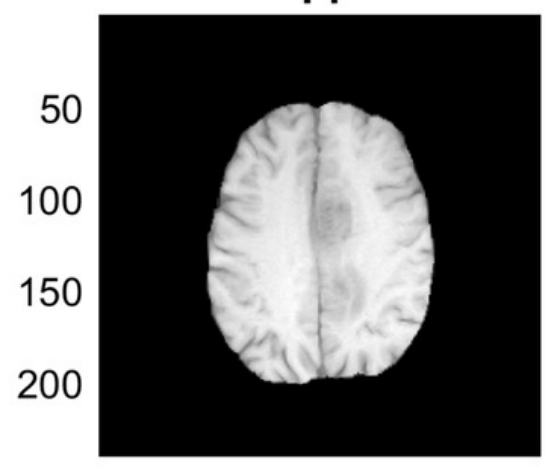

$\begin{array}{llll}50 & 100 \quad 150 \quad 200\end{array}$

(a)

T1C

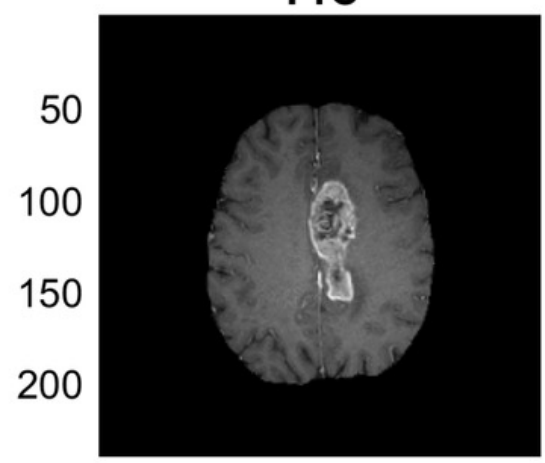

$\begin{array}{llll}50 & 100 & 150 \quad 200\end{array}$

(c)

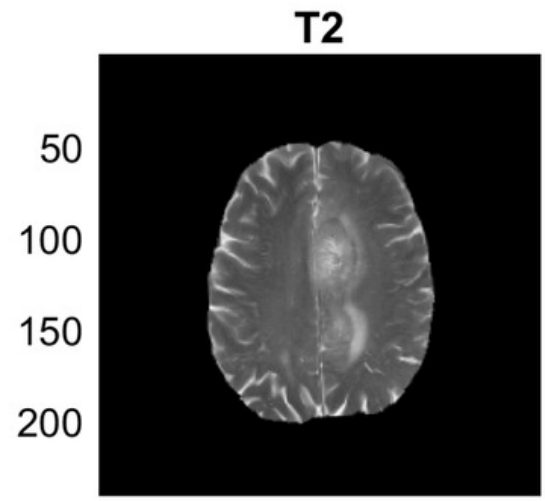

$\begin{array}{llll}50 & 100 \quad 150 \quad 200\end{array}$

(e)

FLAIR

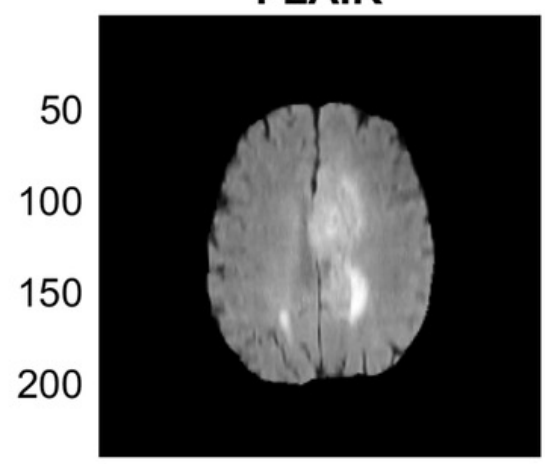

T1

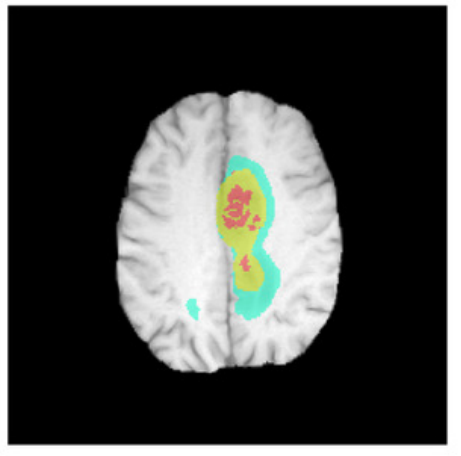

(b)

T1C

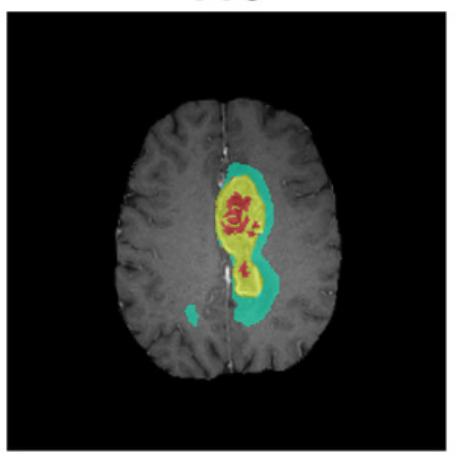

(d)

T2

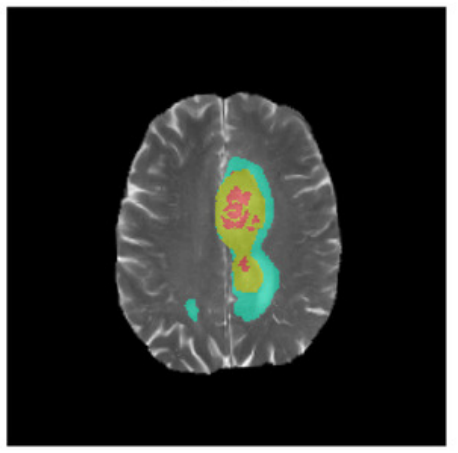

(f)

FLAIR

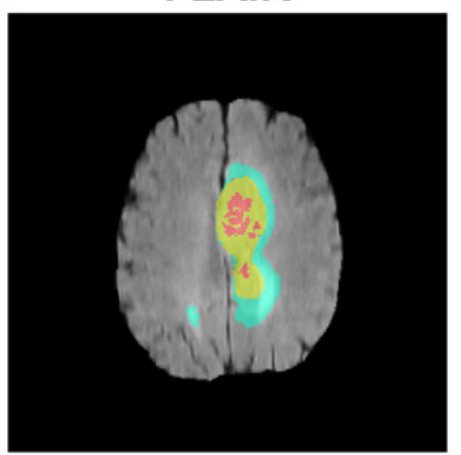

$\begin{array}{llll}50 & 100 & 150 \quad 200\end{array}$

Peer) reviewing(gPF | (2018:08:30103:1:1:NEW 4 Oct 2019/h) 
Figure 3

Performance curves of the five-fold cross validation in the training phase.

(a) shows the ROC for the logistic regression classifier. (b) shows the ROC for the SVM classifier. (c) shows the ROC for the RF classifier.

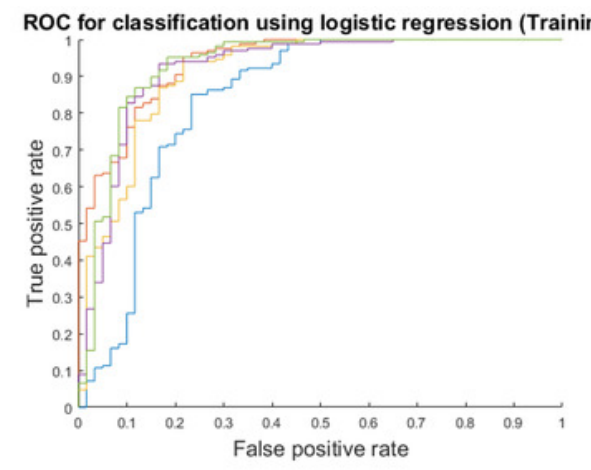

(a)

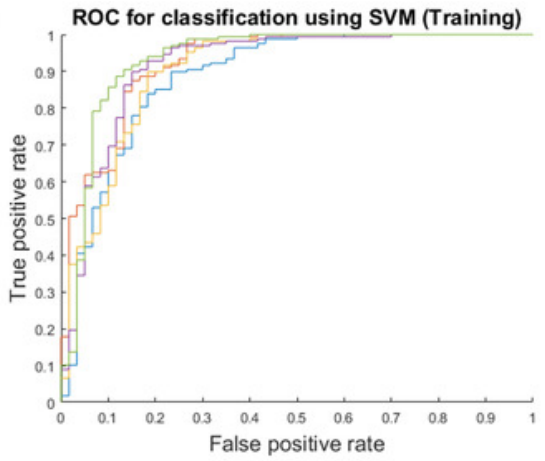

(b)

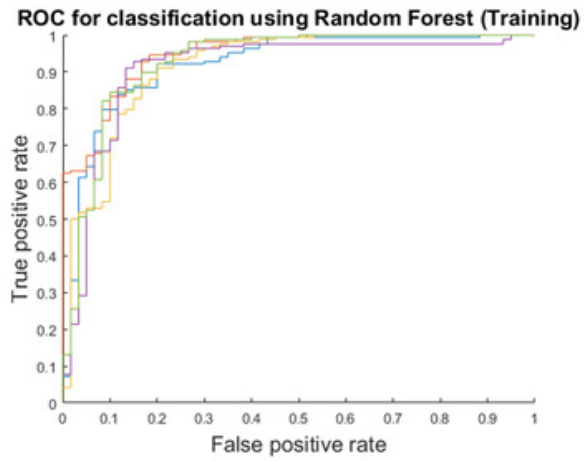

(c) 


\section{Figure 4}

Performance curves of the five-fold cross validation in the test phase.

(a) shows the ROC for the logistic regression classifier. (b) shows the ROC for the SVM classifier. (c) shows the ROC for the RF classifier.

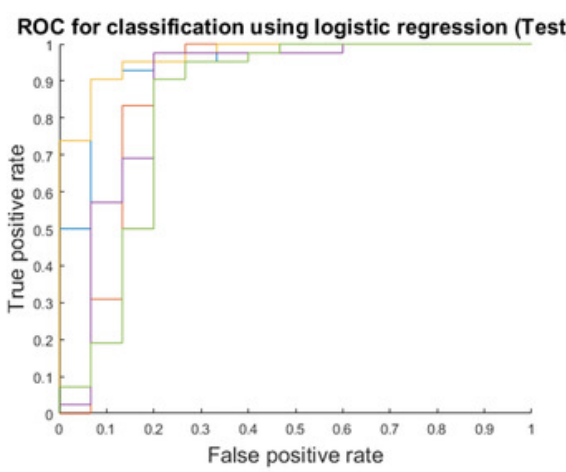

(a)

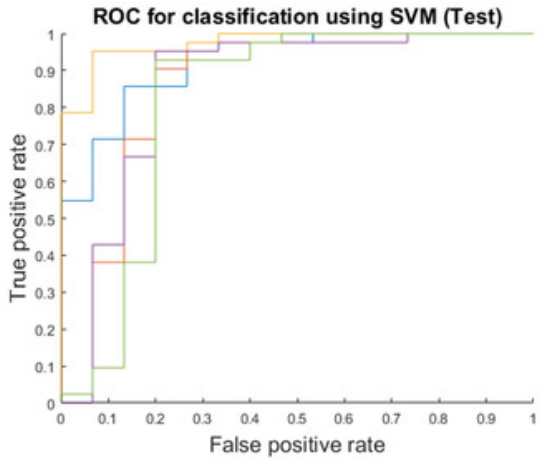

(b)

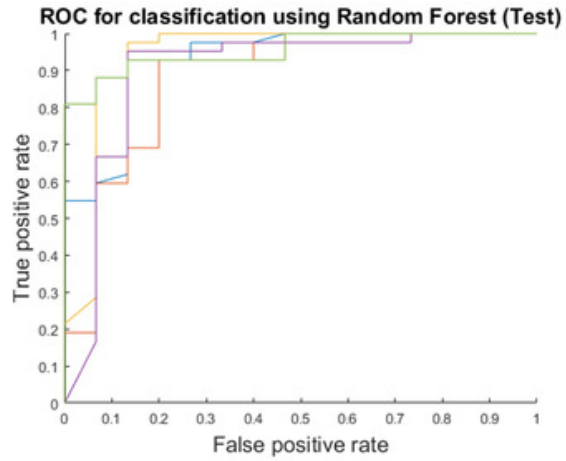

(c) 